\title{
Data adaptive estimation of transversal blood flow velocities
}

\author{
Pirnia, E.; Jakobsson, A.; Gudmundson, E.; Jensen, Jørgen Arendt
}

Published in:

Proceedings of 2014 IEEE International Conference on Acoustics, Speech, and Signal Processing

Link to article, DOI:

10.1109/ICASSP.2014.6854884

Publication date:

2014

Document Version

Early version, also known as pre-print

Link back to DTU Orbit

Citation (APA):

Pirnia, E., Jakobsson, A., Gudmundson, E., \& Jensen, J. A. (2014). Data adaptive estimation of transversal blood flow velocities. In Proceedings of 2014 IEEE International Conference on Acoustics, Speech, and Signal Processing (pp. 6637-6641). IEEE. https://doi.org/10.1109/ICASSP.2014.6854884

\section{General rights}

Copyright and moral rights for the publications made accessible in the public portal are retained by the authors and/or other copyright owners and it is a condition of accessing publications that users recognise and abide by the legal requirements associated with these rights.

- Users may download and print one copy of any publication from the public portal for the purpose of private study or research.

- You may not further distribute the material or use it for any profit-making activity or commercial gain

- You may freely distribute the URL identifying the publication in the public portal 


\title{
DATA ADAPTIVE ESTIMATION OF TRANSVERSAL BLOOD FLOW VELOCITIES
}

\author{
E. Pirnia*, A. Jakobsson*, E. Gudmundson ${ }^{* \dagger}$, and J. A. Jensen ${ }^{\ddagger}$ \\ *Dept. of Mathematical Statistics, Lund University, Sweden \\ ${ }^{\dagger}$ Swedish Defence Research Agency (FOI), Stockholm, Sweden \\ ${ }^{\ddagger}$ Center for Fast Ultrasound Imaging, Technical University of Denmark, Denmark
}

\begin{abstract}
The examination of blood flow inside the body may yield important information about vascular anomalies, such as possible indications of, for example, stenosis. Current medical ultrasound systems suffer from only allowing for measuring the blood flow velocity along the direction of irradiation, posing natural difficulties due to the complex behaviour of blood flow, and due to the natural orientation of most blood vessels. Recently, a transversal modulation scheme was introduced to induce also an oscillation along the transversal direction, thereby allowing for the measurement of also the transversal blood flow. In this paper, we propose a novel data-adaptive blood flow estimator exploiting this modulation scheme. Using realistic Field II simulations, the proposed estimator is shown to achieve a notable performance improvement as compared to current state-of-the-art techniques.
\end{abstract}

Index Terms - Transversal blood velocity estimation, Spectral estimation, BIAA, Medical ultrasound

\section{INTRODUCTION}

The cardiovascular system forms an integral part of our bodies, allowing blood to pulsate through our blood vessels. The pulsatile nature of the blood generates a complex flow pattern within the human body, which may be disrupted by various forms of vascular anomalies, such as stenosis or atherosclerosis. Via medical ultrasound, one is able to diagnose the current state of the cardiovascular system, although current technology is typically restricted to only allow for the estimation of blood flow in the direction of the emitted ultrasound beam, thereby making it difficult to monitor complex flow patterns, or (the commonly occurring) blood vessels perpendicular to the ultrasound beam [1]. One may estimate the blood velocity in the direction of the ultrasound beam, the socalled axial velocity, $v_{z}$, by gathering samples corresponding to the same depth from each pulse emission, producing a signal sampled at the pulse repetition frequency, $f_{p r f}$, which for a single backscatterer would yield a sinusoidal signal with frequency $f_{p}^{v}=2 v_{z} f_{0} / c$, where $v_{z}$ is the blood velocity along

This work was supported in part by the Swedish Research Council and Carl Trygger's foundation. the ultrasound direction, $c=1540 \mathrm{~m} / \mathrm{s}$ the speed of propagation, and $f_{0}$ the emitted ultrasound (centre) frequency (typically 3-10 MHz) [1]. In order to overcome the shortcoming of not being able to estimate velocities perpendicular to the ultrasound beam, the so-called transversal velocity, $v_{x}$, a number of methods have been developed [2-6]. In particular, in the approach proposed in [7] (see also [8-11]), this is achieved by introducing a transversal modulation in the emitted ultrasound pulses, thereby creating an oscillation along the transversal direction of the flow enabling one to use the backscattered signal along this dimension to form an estimate of the transversal velocity. This is done by forming an estimate of the correlation, the power spectral density, or fourth order moment of the sampled signal along the transversal dimension. The method has been extensively evaluated using both simulated flow data and with in vivo data [11-14], and was recently approved by the US FDA [15]. In this work, exploiting the transversal oscillation (TO) technique, we further these works by proposing a novel data-adaptive spectral estimation technique allowing for improved velocity estimates as compared to earlier methods. The proposed algorithm is an extension of the recent BIAA algorithm [16-18] to the TO case. As shown in [16], the BIAA algorithm allows for reliable high-resolution estimates of the axial velocity, and also allows for the use of irregularly sampling schemes, thereby enabling flexibility in the interleaved transmissions used to form the velocity estimation and the B-mode images necessary for navigation inside the vessel; the latter transmissions are currently interleaved between the transmissions used for velocity estimation, thereby halving the effective dynamic span of the velocity estimate (see also [19]). The algorithm presented here, termed the TO-BIAA, shares this benefit, as well as allows for a computationally efficient implementation reminiscent to the one presented in [17].

\section{THE PROPOSED TO-BIAA ALGORITHM}

In conventional colour flow mapping systems, the blood flow velocity estimation is performed by focusing the ultrasound transducer array along a single direction and sampling data at the depth of interest. The signal returned from a monochro- 
Table 1. Parameters for flow simulation

$\begin{array}{lcc}\text { Transducer centre frequency } & f_{0} & 3.42 \mathrm{MHz} \\ \text { Pulse cycles } & \mathrm{M} & 8 \\ \text { Speed of sound } & \mathrm{c} & 1540 \mathrm{~m} / \mathrm{s} \\ \text { Pitch of transducer element } & w & 0.33 \mathrm{~mm} \\ \text { Height of transducer element } & h_{e} & 13 \mathrm{~mm} \\ \text { Kerf } & k_{e} & .0165 \mathrm{~mm} \\ \text { Number of active elements } & N_{e} & 128 \\ \text { Corresponding range gate size } & & 123 \mathrm{~mm} \\ \text { Sampling frequency } & f_{s} & 25 \mathrm{MHz} \\ \text { Pulse repetition frequency } & f_{p r f} & 15 \mathrm{kHz} \\ \text { Radius of vessel } & \mathrm{R} & 4.2 \mathrm{~mm} \\ \text { Distance to vessel center } & Z_{\text {ves }} & 40 \mathrm{~mm}\end{array}$

matic wave at depth $k$ and emission $l$ can then be modelled as $[1,16]$

$$
r_{q}(k, l)=\alpha_{z} \exp \left(j \phi k+j \psi_{z} l\right)+w(k, l),
$$

where $\alpha_{z}$ is the (complex-valued) amplitude, $\phi=2 \pi f_{0} / f_{s}$ and $\psi_{z}=-2 \pi f_{p}^{v} T_{p r f}$, and where $T_{p r f}=1 / f_{p r f}$ and $f_{s}$ is the sampling frequency. Moreover, $w(k, l)$ denotes the noise and any unexplained signal. The axial velocity of the blood flow is therefore proportional to the frequency of the returned signal. However, this technique only allows for estimation of the velocity component along the ultrasound beam direction, $v_{z}$, ignoring the possible flow transverse to the beam direction. As most of the vessels are parallel to the skin surface, techniques allowing for estimation of the transversal blood flow is of great importance. In [7] (see also [8,9]), a transverse oscillation was introduced in the ultrasound beam, influencing the received signal and thus permitting the estimation of both the axial and transverse velocity components. The sampled signal for a monochromatic unit amplitude beam at a given depth may in this case be modelled as [7]

$$
r_{t}(k, l)=\cos \left(\phi k-2 \pi f_{p}^{v} l T_{p r f}\right) \cos \left(2 \pi f_{x}^{v} l T_{p r f}\right),
$$

where $f_{x}^{v}=v_{x} / d_{x}$ and $d_{x}$ indicates the lateral modulation period. In order to obtain a one-sided spectrum, a spatial Hilbert transform of the signal is performed in the direction transverse to the beam, i.e.,

$$
r_{s q}(k, l)=\cos \left(\phi k-2 \pi f_{p}^{v} l T_{p r f}\right) \exp \left(j 2 \pi f_{x}^{v} l T_{p r f}\right) .
$$

The received signal is thus influenced by both the axial and the transversal velocities. In [20], this received signal was used to generate two new signals by taking the temporal Hilbert transform of (3), but this time in the direction of the beam, i.e.,

$$
r_{s q h}(k, l)=\sin \left(\phi k-2 \pi f_{p}^{v} l T_{p r f}\right) \exp \left(j 2 \pi f_{x}^{v} l T_{p r f}\right) .
$$

Using Euler's formula, (3) and (4) may be rewritten as

$$
\begin{aligned}
r_{s q}(k, l)= & \frac{1}{2} \exp \left[j 2 \pi\left(f_{x}^{v}-f_{p}^{v}\right) l T_{p r f}+j \phi k\right]+ \\
& \frac{1}{2} \exp \left[j 2 \pi\left(f_{x}^{v}+f_{p}^{v}\right) l T_{p r f}-j \phi k\right], \\
r_{s q h}(k, l)= & \frac{1}{2 j} \exp \left[j 2 \pi\left(f_{x}^{v}-f_{p}^{v}\right) l T_{p r f}+j \phi k\right]- \\
& \frac{1}{2 j} \exp \left[j 2 \pi\left(f_{x}^{v}+f_{p}^{v}\right) l T_{p r f}-j \phi k\right] .
\end{aligned}
$$

Combining (5) and (6), two new signals $r_{1}$ and $r_{2}$ may thus be composed as

$$
\begin{aligned}
r_{1}(k, l) & =r_{s q}(k, l)+j r_{s q h}(k, l) \\
& =\exp \left[j\left(2 \pi\left(f_{p}^{v}+f_{x}^{v}\right) l T_{p r f}+\phi k\right)\right], \\
r_{2}(k, l) & =r_{s q}(k, l)-j r_{s q h}(k, l) \\
& =\exp \left[j\left(2 \pi\left(f_{x}^{v}-f_{p}^{v}\right) l T_{p r f}-\phi k\right)\right] .
\end{aligned}
$$

Using these, the combined signal $r_{1}(k, l) r_{2}(k, l)$ may thus be used to form an estimate of the transversal velocity component, i.e.,

$$
r_{12}(k, l)=r_{1}(k, l) r_{2}(k, l)=\exp \left(j \psi_{x} l\right),
$$

where $\psi_{x}=2 \pi\left(2 f_{x}^{v}\right) T_{p r f}=2 \pi T_{p r f}\left(2 v_{x} / d_{x}\right)$. It may be noted that the transverse blood flow velocity is thus proportional to the frequency of $r_{12}(k, l)$. We proceed to model $r_{12}(k, l)$ as a signal constructed as a sum of contributions from all considered frequency grid points, $\left\{\psi_{m, x}\right\}_{m=1}^{M}$, i.e.,

$$
r_{12}(k, l)=\sum_{m=1}^{M} \alpha_{m, x}^{(k)} \exp \left(j \psi_{m, v_{x}}\right)+e(k, l),
$$

where $\alpha_{m, x}^{(k)}$ denotes the (complex-valued) amplitude at depth $k$, and $e(k, l)$ is a zero mean white complex Gaussian noise, thus implying that any noise colour in the measurement of $r_{12}(k, l)$ is modelled as contributing to the signal via $\alpha_{x}^{(k)}$. It is worth noting that this model does not impose any assumptions on the used sampling patterns over the depth or the emissions. Due to smoothness of the blood flow profile, the blood spectral amplitude over a range of depths, $\alpha_{m, x}^{(k)}, k=$ $k_{1}, \ldots, k_{K}$, will be almost constant as long as the range of considered depth is limited to be within the emitted pulse length. Reminiscent of [16], we thus introduce

$$
\begin{aligned}
\mathbf{r}_{k} & =\left[\begin{array}{lll}
r_{12}\left(k, l_{1}\right) & \ldots & r_{12}\left(k, l_{N}\right)
\end{array}\right]^{T}, \\
\boldsymbol{\alpha}_{x}^{(k)} & =\left[\begin{array}{lll}
\alpha_{1, x}^{(k)} & \ldots & \alpha_{M, x}^{(k)}
\end{array}\right]^{T}, \\
\mathbf{A} & =\left[\begin{array}{lll}
\mathbf{a}_{1} & \ldots & \mathbf{a}_{M}
\end{array}\right], \\
\mathbf{a}_{m} & =\left[\begin{array}{lll}
\exp \left(j \psi_{m, x} l_{1}\right) & \ldots & \exp \left(j \psi_{m, x} l_{N}\right)
\end{array}\right]^{T},
\end{aligned}
$$

where ${ }^{T}$ denotes the transpose, allowing (10) to be expressed as

$$
\mathbf{r}_{k}=\mathbf{A} \boldsymbol{\alpha}_{x}^{(k)}+\mathbf{e}_{k},
$$




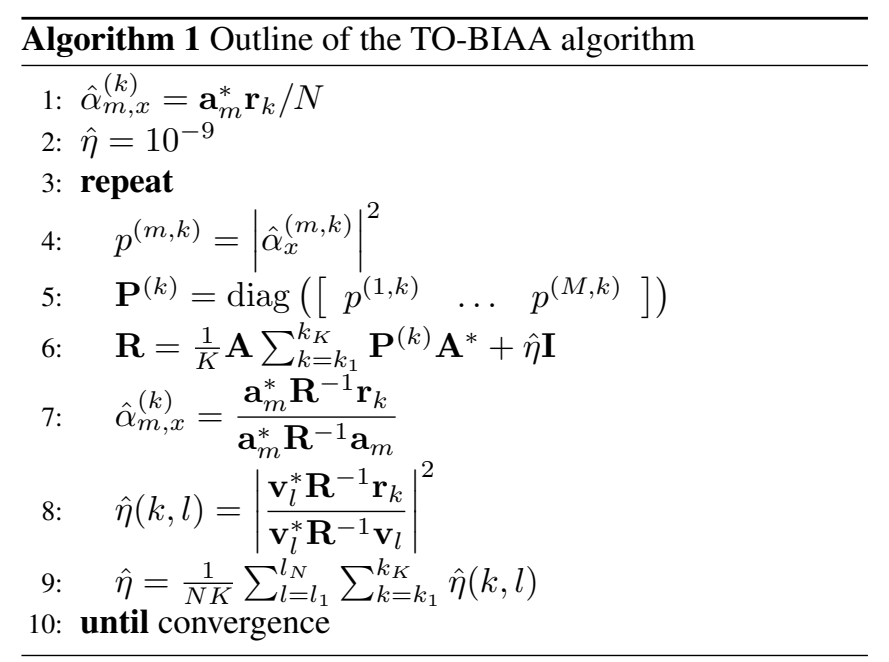

where $\mathbf{e}_{k}$ is defined similarly to $\mathbf{r}_{k}$. The covariance matrix of $\mathbf{r}_{k}$ may thus be expressed as

$$
\mathbf{R}^{(k)}=\sum_{m=1}^{M}\left|\alpha_{m, x}^{(k)}\right|^{2} \mathbf{a}_{m} \mathbf{a}_{m}^{*}+\eta \mathbf{I}=\mathbf{A} \mathbf{P}^{(k)} \mathbf{A}^{*}+\eta \mathbf{I}
$$

where * and I denote the Hermitian transpose and the identity matrix, respectively, and

$$
\begin{aligned}
\mathbf{P}^{(k)} & =\operatorname{diag}\left(\left[\begin{array}{lll}
p^{(1, k)} & \ldots & p^{(M, k)}
\end{array}\right]\right), \\
p^{(m, k)} & =\left|\alpha_{m, x}^{(k)}\right|^{2}
\end{aligned}
$$

with $\operatorname{diag}(\mathbf{x})$ denoting a matrix which has the elements of the vector $\mathbf{x}$ along its diagonal. Averaging the covariance matrix over neighbouring depths yields

$$
\mathbf{R}=\frac{1}{K} \sum_{k=k_{1}}^{k_{K}} \mathbf{R}^{(k)}=\frac{1}{K} \mathbf{A}\left[\sum_{k=k_{1}}^{k_{K}} \mathbf{P}^{(k)}\right] \mathbf{A}^{*}+\eta \mathbf{I} .
$$

In order to estimate $\alpha_{m, x}^{(k)}$ using $\mathbf{R}$, we form the matchedfilterbank amplitude estimate as (cf. [16,21])

$$
\hat{\alpha}_{m, x}^{(k)}=\frac{\mathbf{a}_{m}^{*} \mathbf{R}^{-1} \mathbf{r}_{k}}{\mathbf{a}_{m}^{*} \mathbf{R}^{-1} \mathbf{a}_{m}} .
$$

By then averaging over neighbouring depths, one may estimate amplitude in the centre of the vessel as

$$
\hat{\alpha}_{m, v_{x}}=\frac{1}{K} \sum_{k=k_{1}}^{k_{K}} \hat{\alpha}_{m, x}^{(k)}
$$

Similarly, an estimate of the noise variance at depth $k$, for emission $\ell$, may be formed as

$$
\hat{\eta}(k, l)=\left|\frac{\mathbf{v}_{l}^{*} \mathbf{R}^{-1} \mathbf{r}_{k}}{\mathbf{v}_{l}^{*} \mathbf{R}^{-1} \mathbf{v}_{l}}\right|^{2},
$$

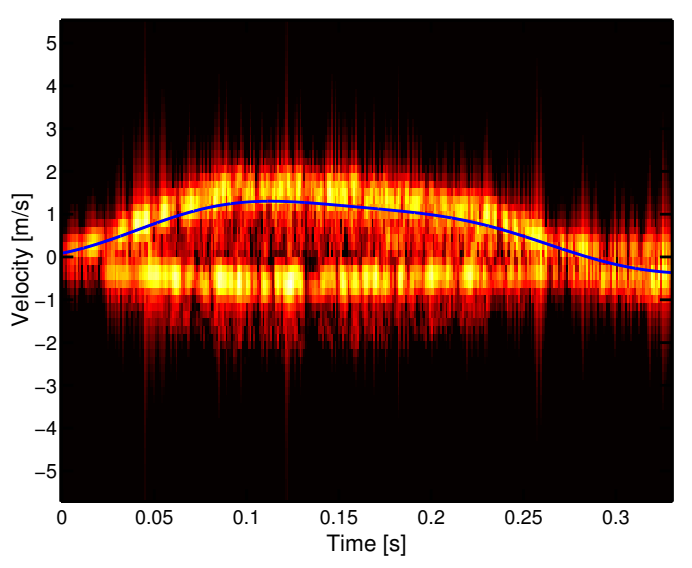

(a) 2nd-order method.

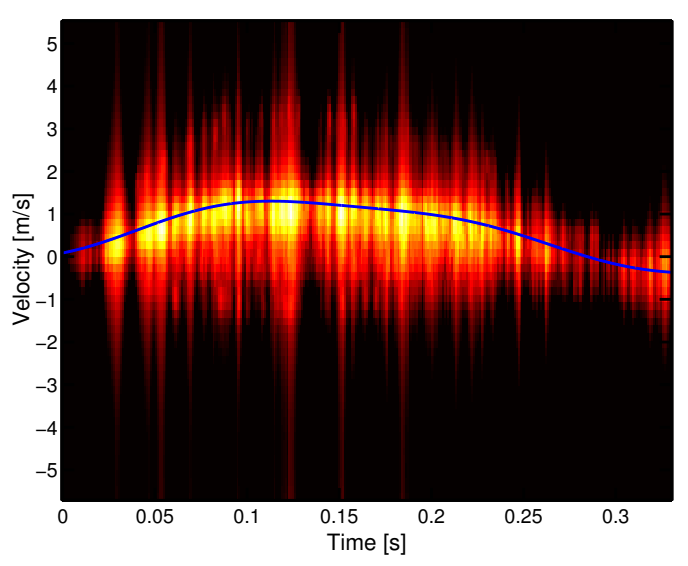

(b) 4th-order method.

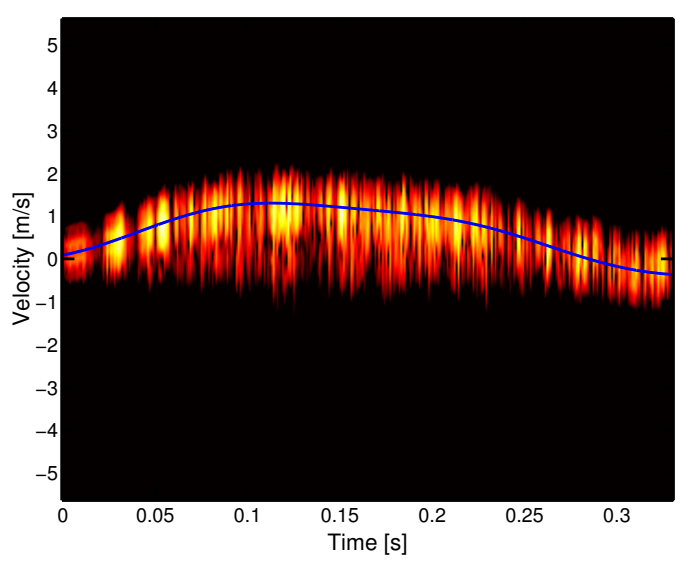

(c) TO-BIAA

Fig. 1. Transversal blood velocity estimates from 32 emissions using (a) the 2nd-order method, (b) the 4th-order method, and (c) TO-BIAA, as compared to the expected mean velocity (blue line). 
where $\mathbf{v}_{\ell}$ denotes the $\ell$ th column of $\mathbf{I}$. The averaged noise variance is then found as

$$
\hat{\eta}=\frac{1}{N K} \sum_{l=l_{1}}^{l_{N}} \sum_{k=k_{1}}^{k_{K}} \hat{\eta}(k, l) .
$$

It should be noted that as $\mathbf{R}$ depends on $\alpha_{m, x}$, the proposed TO-BIAA method is implemented using an iterative algorithm. The outline of this algorithm is shown in Algorithm 1; the algorithm is here initiated using a least squares estimate, and is then iterated until practical convergence, which generally requires no more than $10-15$ iterations.

\section{NUMERICAL RESULTS}

We proceed to evaluate the proposed TO-BIAA using realistic blood flow data generated using the Field II toolbox [22], mimicking the blood flow in a femoral artery. Figures 1 and 2 illustrate the achieved performance of the proposed estimator as compared to the 2 nd-order and the 4th-order methods, where the cross-correlation of (7) and (8), and the product of the auto-correlations of (7) and (8), respectively, are used to form the velocity estimate (see $[7,15,20]$ for details on these algorithms). As is clear from the figures, the 2nd-order method suffers from strong spectral artefacts whereas the 4thorder method has problems with yielding broad spectral lines. In comparison, TO-BIAA shows no artefacts and less spectral broadening. Here, the Womersley model [23] for pulsating flow was used and the specific parameters for the flow simulation are summarised in Table 1. As is typically done, the stationary part of the signal was removed by mean subtraction. Moreover, all spectrograms were produced using a dynamic range of $30 \mathrm{~dB}$, and the velocity was estimated in the centre of vessel, using 30 samples along depth. In total, 32 and 64 emissions, respectively, were used to generate the spectrum, and the angle of propagation was set to $75^{\circ}$, where $90^{\circ}$ corresponds to a beam orthogonal to the blood vessel. Comparing Figures 1 and 2, it may be noted that the additional emissions help focusing the TO-BIAA estimate noticeably, whereas the other estimators still suffer from poor performance.

\section{CONCLUSION}

In this work, we have, using a transversal modulation in the ultrasound pulse emissions, exploited the the resulting transversal oscillation to form a data-adaptive blood flow estimator, allowing for the estimation of also transversal velocities. The proposed estimator is an extension of the recent BIAA blood flow estimator, and is, as the BIAA estimator, able to form reliable estimates even for non-uniformly sampled emission schemes. Numerical illustrations show the performance of the proposed method as compared to existing techniques.

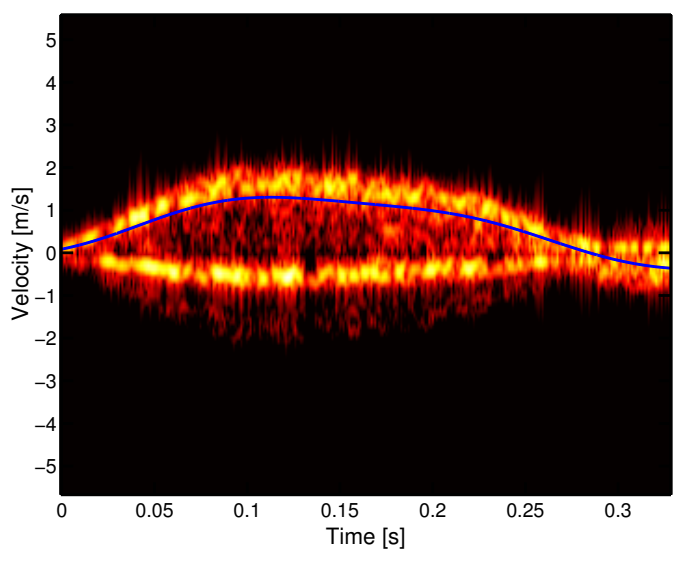

(a) 2nd-order method.

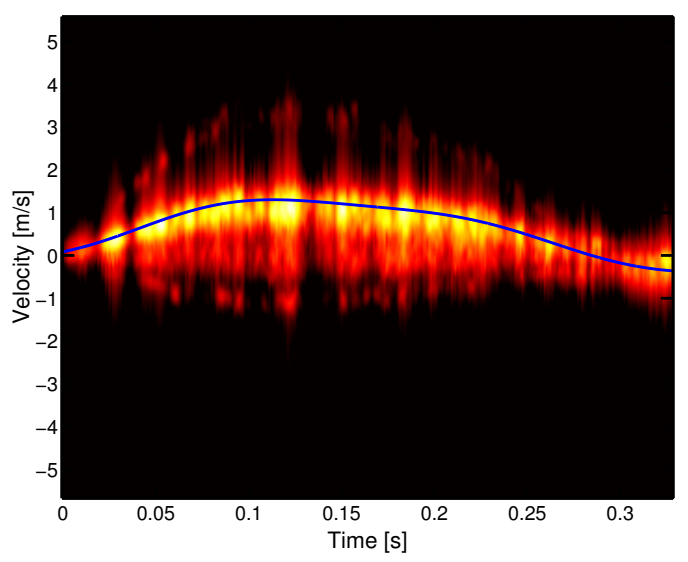

(b) 4th-order method.

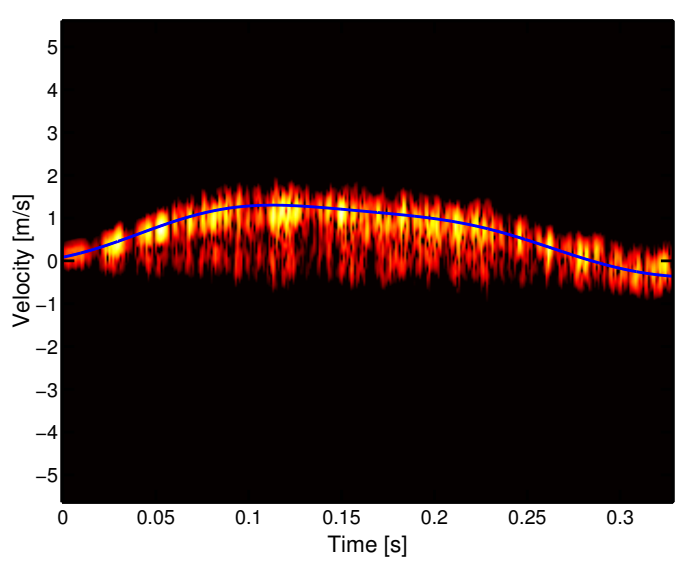

(c) TO-BIAA

Fig. 2. Transversal blood velocity estimates from 64 emissions using (a) the 2nd-order method, (b) the 4th-order method, and (c) TO-BIAA, as compared to the expected mean velocity (blue line). 


\section{REFERENCES}

[1] J. A. Jensen, Estimation of Blood Velocities Using Ultrasound. New York: Cambridge University Press, 1996.

[2] M. D. Fox, "Multiple crossed-beam ultrasound Doppler velocimetry," IEEE Trans. Sonics Ultrason., vol. 25, no. 5, pp. 281-286, 1978.

[3] G. E. Trahey, J. W. Allison, and O. T. van Ramm, "Angle independent ultrasonic detection of blood flow," IEEE Trans. Biomed. Eng., vol. 34, pp. 965-967, 1987.

[4] V. L. Newhouse, D. Censor, T. Vontz, J. A. Cisneros, and B. B. Goldberg, "Ultrasound Doppler probing of flows transverse with respect to beam axis," IEEE Trans. Biomed. Eng., vol. 34, pp. 779-788, 1987.

[5] O. Bonnefous, "Measurement of the complete (3D) velocity vector of blood flows," in Proc. IEEE Ultrason. Symp., (Chicago, IL, USA), pp. 795-799, 1988.

[6] J. A. Jensen, "Directional velocity estimation using focusing along the flow direction. I: Theory and simulation," IEEE Trans. Ultrason., Ferroelectr., Freq. Control, vol. 50, no. 7, pp. 857-872, 2003.

[7] J. A. Jensen, "Spectral Velocity Estimation in the Transverse Direction," in Proc. IEEE Intern. Ultrason. Symp. (IUS), (Prague, Czech Republic), 2013.

[8] J. A. Jensen and P. Munk, "A new method for estimation of velocity vectors," IEEE Trans. Ultrason., Ferroelectr., Freq. Control, vol. 45, pp. 837-851, May 1998.

[9] J. A. Jensen, "A new estimator for vector velocity estimation," IEEE Trans. Ultrason., Ferroelectr., Freq. Control, vol. 48, no. 4, pp. 886-894, 2001.

[10] M. Anderson, "Multi-dimensional velocity estimation with ultrasound using spatial quadrature," IEEE Trans. Ultrason., Ferroelectr., Freq. Control, vol. 45, pp. 852 -861, May 1998.

[11] J. Udesen and J. A. Jensen, "Investigation of transverse oscillation method," IEEE Trans. Ultrason., Ferroelectr., Freq. Control, vol. 53, pp. 959-971, May 2006.

[12] K. L. Hansen, J. Udesen, N. Oddershede, L. Henze, C. Thomsen, J. A. Jensen, and M. B. Nielsen, "In vivo comparison of three ultrasound vector velocity techniques to MR phase contrast angiography," Ultrasonics, vol. 49, no. 8, pp. 659-667, 2009.

[13] K. Hansen, J. Udesen, C. Thomsen, J. A. Jensen, and M. Nielsen, "In vivo validation of a blood vector velocity estimator with MR angiography," IEEE Trans. Ultrason., Ferroelectr., Freq. Control, vol. 56, no. 1, pp. 91100, 2009.
[14] M. M. Pedersen, M. J. Pihl, P. Haugaard, J. M. Hansen, K. L. Hansen, M. B. Nielsen, and J. A. Jensen, "Comparison of Real-Time In Vivo Spectral and Vector Velocity Estimation," Ultrasound Med. Biol., vol. 38, no. 1, pp. 145-151, 2012.

[15] J. Jensen, M. Pihl, J. Olesen, P. Møller Hansen, K. Lindskov Hansen, and M. Bachmann Nielsen, "New Developments in Vector Velocity Imaging Using the Transverse Oscillation Approach," in Proc. SPIE, vol. 8675, 2013.

[16] E. Gudmundson, A. Jakobsson, J. A. Jensen, and P. Stoica, "Blood Velocity Estimation Using Ultrasound and Spectral Iterative Adaptive Approaches," Signal Process., vol. 91, pp. 1275-1283, May 2011.

[17] G.-O. Glentis and A. Jakobsson, "Efficient Implementation of Iterative Adaptive Approach Spectral Estimation Techniques," IEEE Trans. Signal Process., vol. 59, pp. 4154-4167, Sept. 2011.

[18] A. Jakobsson, G. O. Glentis, and E. Gudmundson, "Computationally Efficient Time-Recursive IAA-Based Blood Velocity Estimation," IEEE Trans. Signal Process., vol. 60, July 2012.

[19] E. Gudmundson, A. Jakobsson, and F. Gran, "Overcoming the Nyquist Limit in Blood Flow Velocity Estimation," in Proc. IEEE Intern. Ultrason. Symp. (IUS), (Dresden, Germany), Oct. 2012.

[20] J. A. Jensen and P. Munk, "An improved estimation and focusing scheme for vector velocity estimation," in Proc. IEEE Ultrason. Symp., vol. 2, pp. 1465-1470, 1999.

[21] P. Stoica, A. Jakobsson, and J. Li, "Matched-Filterbank Interpretation of Some Spectral Estimators," Signal Process., vol. 66, pp. 45-59, April 1998.

[22] J. A. Jensen and N. B. Svendsen, "Calculation of pressure fields from arbitrarily shaped, apodized, and excited ultrasound transducers," IEEE Trans. Ultrason., Ferroelectr., Freq. Control, vol. 39, pp. 262-267, 1992.

[23] J. R. Womersley, "Oscillatory motion of a viscous liquid in a thin-walled elastic tube. I: The linear approximation for long waves," Phil. Mag., vol. 46, pp. 199-221, 1955. 